\title{
ORIGINAL
}

\section{VALIDEZ DE DISTINTAS MEDIDAS DE CONSUMO DE TABACO DURANTE EL EMBARAZO: ESPECIFICIDAD, SENSIBILIDAD Y PUNTOS DE CORTE DÓNDE Y CUÁNDO (*)}

\author{
Jose Manuel Aranda Regules (1), Pedro Mateos Vilchez (2), Asunción González Villalba (3), \\ Fuensanta Sanchez (4) y Juan de Dios Luna del Castillo (5)
}

(1) Escuela Andaluza de Salud Pública. Granada.

(2) Equipo de Salud Mental "Las Albarizas" Marbella Málaga.

(3) Plan Integral de Tabaquismo de Andalucía. Málaga.

(4) Centro de Salud el Palo Málaga.

(5) Facultad de Medicina de Granada.

\section{RESUMEN}

Fundamentos: La ocultación del hábito tabáquico en mujeres embarazadas fumadoras cuestiona la validez de la autodeclaración Los objetivos de esta investigación son determinar el índice de ocultación y establecer la validez del $\mathrm{CO}$ en aire espirado como método de validación bioquímica.

Método: Datos obtenidos de un Ensayo Clínico Aleatorizado en 12 Centros de Salud de Málaga. 454 mujeres embarazadas fumadoras de las cuales manifestaron abandonar 104 al inicio. Medición del habito: autodeclaración, monóxido de carbono (CO) y cotinina en orina en las que declararon no fumar. Para el CO se obtuvo la curva ROC calculándose el área bajo la curva y la sensibilidad y especificidad para diferentes puntos de corte. Empleando el CO como patrón oro se determinó la validez de la autodeclaración, en términos de la sensibilidad y la especificidad.

Resultados: Comparación cotinina/autodeclaración: tasa de ocultación 15,4\% (IC 95\% 9,3-24,1). Comparación cotinina/CO: Área bajo la curva ROC de 0,838 (IC 95\% 0,740-0,935). Para punto de corte de 9 , recomendado en la bibliografía, logramos especificidad $100 \%$ con sensibilidad 12,5\%. Comparación CO/autodeclaración (punto de corte 9): Falsos negativos $0.8 \%$, prevalencia abandono espontáneo $58,1 \%$.

Conclusiones: Se confirma un porcentaje de ocultación similar a otros estudios y la validez del CO como método para la identificación de mujeres fumadoras. En punto de corte 9, la validez de $\mathrm{CO}$ y autodeclaración serían similares, aumentando hasta cifras irreales la prevalencia del abandono espontáneo En la actualidad no hay pruebas suficientes para recomendar el 9 como punto de corte de CO. Los resultados sugieren que este debería ser inferior. Son necesarias investigaciones más amplias en mujeres gestantes que se declaren no fumadoras con una metodología adecuada

Palabras clave: Embarazo. Tabaquismo. Estudios de validación.

Correspondencia:

Jose Manuel Aranda Regules

AV/ del Pacifico, 160

Urbanización Montealto $1^{\text {a }}$ fase.

Arroyo de la Miel, Benalmádena. 29639 Málaga

Correo electrónico: regules11@gmail.com

\section{ABSTRACT \\ Validity of Smoking Measurements during Pregnancy: Specificity, Sensitivity and Cut-Off Points}

Background: Non disclosure by pregnant women smokers of their smoking habit questions the validity of self-declarations. The purpose of this research is to determine the rate of Non disclosure and to establish the validity of exhaled $\mathrm{CO}$ as a method of biochemical validation.

Method: Data obtained in a Randomised Clinical Trial in 12 Health Centres in Malaga. 454 pregnant women smokers, of whom 104 declared that they had stopped smoking at the start. Measurement of the habit: self-declaration, carbon monoxide $(\mathrm{CO})$ and cotinine in the urine in those subjects who declared that they no longer smoked. The ROC curve was obtained for the CO, calculating the area under the curve and the sensitivity and specificity for different cut-off points. Using the $\mathrm{CO}$ as the gold standard, the validity of the selfdeclarations was determined in terms of sensitivity and specificity.

Results: Cotinine/self-declaration comparison: rate of Non disclosure 15.4\% (IC 95\% 9.3-24.1). Cotinine/CO comparison: Area under the ROC curve of 0.838 (IC 95\% 0.740-0.935). For a cut-off point of 9 , recommended in the relevant bibliography, we achieved $100 \%$ specificity with $12.5 \%$ sensitivity. CO/self-declaration comparison (cut-off point 9): False negations $0.8 \%$, prevalence of spontaneous abandonment of smoking habit $58.1 \%$.

Conclusions: A percentage of Non disclosure similar to other studies and the validity of $\mathrm{CO}$ as a method for the identification of women smokers are confirmed. At cut-off point 9, the validity of $\mathrm{CO}$ and self-declaration would be similar, with the prevalence of spontaneous abandonment increasing up to unreal figures. At the present time, the evidence is not sufficient in order to recommend 9 as the CO cut-off point. The results suggest that this should be lower. More extensive research is necessary in pregnant women who state that they do not smoke, using an appropriate methodology.

Key words: Pregnancy. Tobacco. Validation studies.

(*) Esta investigación ha sido financiada por el Plan de Investigación de la Conserjería de Salud de la Junta de Andalucía en su convocatoria anual del año 2001. 


\section{INTRODUCCIÓN}

Tanto los estudios de prevalencia de tabaquismo en el embarazo, como los de eficacia y efectividad están sometidos al posible sesgo de la ocultación/engaño sobre los hábitos de consumo. Este problema puede ser explicado por la presión social a la que están expuestas las embarazadas fumadoras que lleva, en muchos casos, a que mantenga su hábito tabáquico de manera clandestina y secreta.

El autoinforme de consumo se viene utilizando de manera sistemática para valorar el estatus fumador, la cantidad de cigarrillos fumados y/o la exposición al humo de tabaco de los fumadores habituales, tanto en estudios poblacionales como en ensayos clínicos. Si bien este índice se considera como una medida aceptable que refleja con relativa exactitud el consumo/exposición directa al tabaco en la población general en nuestro medio ${ }^{1}$, no se puede decir lo mismo en embarazadas fumadoras ${ }^{2}$. Aunque los porcentajes de ocultación pueden minimizarse con cuestionarios que no exploren de forma directa el hábito, en el artículo de Russell et al ${ }^{3}$ se pone de manifiesto que este índice, en los 15 estudios revisados que cumplían criterios de calidad y contaban con algún tipo de validación bioquímica de la autodeclaración, osciló entre el $0 \%$ y el $48 \%$. Hay que resaltar la gran variabilidad del fenómeno que depende no solo del estudio (y por tanto de las características de la intervención y los terapeutas), sino del momento de la gestación en que se realizo la medición y de la condición experimental de cada sujeto.

En definitiva, siguiendo a Windsor et al. ${ }^{4}$, el informe verbal de la gestante debería ser validado mediante determinaciones bioquímicas de sustancias que sirvan como índices objetivos del consumo/exposición al tabaco, ya que no se puede considerar que la autodeclara- ción sea precisa, debido a que está sujeta a un grado de ocultación. De no hacerlo, supondría la subestimación de la prevalencia de tabaquismo, así como la sobrestimación de las tasas de abandono/reducción, con sus consecuencias tanto en la evaluación de programas de intervención como en los estudios epidemiológicos de prevalencia en esta población.

Las medidas bioquímicas que más se utilizan para validar la autodeclaración son el monóxido de carbono en el aire espirado y la cotinina en saliva u orina ${ }^{56}$.

La cotinina puede considerarse el "patrón oro" por la capacidad para detectar el consumo bajo o esporádico de tabaco ${ }^{7}$, pero tiene el inconveniente fundamental de su poca accesibilidad y alto coste. La otra medición que se ha utilizado en los estudios con gestantes es el monóxido de carbono en aire espirado $(\mathrm{CO})^{8}$. Sin embargo, esta medida de corte fisiológico tiene limitaciones. Así, la vida media del $\mathrm{CO}$ es corta, entre 1 y 4 horas $^{9}$ ) y por tanto no puede ser usada para detectar exposición activa al humo de tabaco previa a las 24 horas de la medición. Otros factores que influyen en las determinaciones de $\mathrm{CO}$ en embarazadas, independientemente del consumo activo de cigarrillos, son el efecto acumulado de otras exposiciones (humo de otros fumadores), el consumo previo al embarazo, la edad gestacional $^{10}$, el tiempo que ha pasado desde el último cigarrillo fumado y la marca de cigarrillos, aunque de estas influencias solo se mantiene al final del embarazo el tiempo trascurrido desde el ultimo cigarrillo ${ }^{11}$. La hora del día a la que se hace la medición y el medio donde se vive (gases de automóviles, contaminación, etc.) también han sido citados como factores distorsionantes. También en este sentido, se tienen pruebas de que la absorción y la eliminación de este gas varían de unos fumadores a otros en función de su grado de actividad física y la presencia o ausencia de enfermedades pulmonares $^{12,13}$. 
Los valores de $\mathrm{CO}$ que permiten detectar si la gestante ha fumado o no, oscilan en los distintos estudios de intervención con gestantes fumadoras que utilizan este método de validación bioquímica. El rango va desde 5 p.p.m. ${ }^{14}$, pasando por $7^{15,16}$ hasta 8 p.p.m. ${ }^{17,18}$. Solamente los estudios de Hartman ${ }^{14}$ y Hugues ${ }^{15}$ determinaron sus puntos de corte con datos obtenidos de población gestante fumadora.

En consecuencia, con la investigación cuyos resultados presentamos pretendíamos:

1. Determinar cual es el índice de ocultación/engaño de la autodeclaración del consumo de tabaco en gestantes fumadoras que declaran no fumar en nuestro medio cuando se compara con los valores de cotinina en orina considerada como "patrón oro".

2. Establecer en nuestra población la validez de la determinación del $\mathrm{CO}$ en aire espirado en comparación con la determinación de cotinina en orina, así como los puntos de corte para diferenciar con el menor margen de error posible entre gestantes fumadoras y no fumadoras. Si encontramos que la validez de este último método es aceptable y conocida podremos sustituir una prueba de alto coste y escasa accesibilidad por otra de bajo coste y fácil disponibilidad para el trabajo clínico y la investigación.

\section{SUJETOS Y MÉTODO}

Este estudio forma parte de un ensayo clínico aleatorizado diseñado para evaluar la efectividad de tres intervenciones de intensidad creciente para promover el abandono del hábito tabáquico durante la gestación, integradas en el programa de atención prenatal del Sistema Sanitario Público de Andalucía. Se ha desarrollado en 12 Centros de Salud de Atención Primaria del Dis- trito Sanitario de Málaga que fueron asignados a través de un listado de números aleatorios, a los brazos del estudio, entre los años 2001-2004. Ninguno de los participantes en el proceso de asignación perteneció al equipo investigador, ni intervino en el estudio en ningún momento.

La muestra estaba constituida por 454 mujeres embarazadas fumadoras de al menos 1 cigarrillo diario en el momento del diagnostico del embarazo, que asistieron a la primera visita de control (cobertura del programa $80 \%$ del total de embarazos en el área). La descripción de la muestra aparece la tabla 1. El tamaño de muestra de este ensayo se calculó para un error alfa de un $5 \%$ y una potencia en la comparación más extrema de un $80 \%$, suponiendo que la correlación intraclase sea de un $0.025^{19}$ Las participantes fueron cegadas para el tipo de intervención y eran captadas antes de la semana 15 de gestación en las visitas del programa de Control de Embarazo por parte de una matrona con formación y entrenamiento en esta labor. Tras solicitar el pertinente consentimiento informado y cumplimentar una ficha para el seguimiento, se la invitaba a una entrevista personal con un investigador independiente del terapeuta.. De manera específica, por lo que respecta a los resultados que presentamos, se obtuvo, al inicio del embarazo, información verbal acerca del consumo de tabaco con un cuestionario indirecto, así como una medición de las concentraciones de monóxido de carbono en aire espirado (determinado mediante el cooxímetro Bedfont ${ }^{\circledR}$ micro smokerlyzer) y una muestra de orina para determinaciones de los niveles de cotinina solo en las gestantes que declararon no fumar. Las muestras de orina para las mediciones de cotinina se congelaron a - $4 \mathrm{C}^{\circ}$ para remitir al laboratorio donde se determinaron los niveles de cotinina mediante radioinmunoensayo. Se estableció un punto de corte de 100 ng/ml como nivel mínimo para descartar tabaquismo activo. 
Tabla 1

Descriptiva de la muestra de gestantes fumadoras. Distrito Sanitario Málaga. 2001

\begin{tabular}{|c|c|c|c|}
\hline \multicolumn{2}{|l|}{ Variables } & \multirow{2}{*}{$\begin{array}{c}\text { Casos } \\
100 \\
\end{array}$} & \multirow{2}{*}{$\begin{array}{l}\% \\
22,0\end{array}$} \\
\hline Edad (años) & $<25$ & & \\
\hline & $25-35$ & 304 & 66,8 \\
\hline & $>35$ & 50 & 11,0 \\
\hline \multirow[t]{4}{*}{ Nivel de estudios } & Primer grado & 19 & 4,2 \\
\hline & Secundaria 1er, Ciclo & 205 & 45,1 \\
\hline & Secundaria $2^{\circ}$ Ciclo & 153 & 33,6 \\
\hline & Tercer grado & 78 & 17,1 \\
\hline \multirow{4}{*}{$\begin{array}{l}\text { Ocupación/clase } \\
\text { Social (nivel) }\end{array}$} & Alto & 22 & 4,8 \\
\hline & Medio-alto & 112 & 24,6 \\
\hline & Medio-bajo & 243 & 53,4 \\
\hline & Bajo & 78 & 17,1 \\
\hline \multirow{4}{*}{$\begin{array}{l}\text { Edad Gestacional en el momento de la Entrevista } \\
\text { (semanas) }\end{array}$} & 9 & 131 & 28,8 \\
\hline & $10-12$ & 118 & 25,9 \\
\hline & 13-15 & 101 & 22,2 \\
\hline & $>15$ & 105 & 23,1 \\
\hline \multirow[t]{3}{*}{ Paridad } & 1 & 259 & 56,9 \\
\hline & 2 & 155 & 34,1 \\
\hline & 3 ó más & 41 & 9,0 \\
\hline \multirow{6}{*}{$\begin{array}{l}\text { Consumo diario (cigarrillos) en el momento de la } \\
\text { entrevista }\end{array}$} & 0 & 135 & 29,7 \\
\hline & $1-5$ & 156 & 34,3 \\
\hline & $5-10$ & 107 & 23,5 \\
\hline & $11-15$ & 31 & 6,7 \\
\hline & $16-20$ & 20 & 4,4 \\
\hline & $>20$ & 6 & 1,3 \\
\hline \multicolumn{2}{|l|}{ Casos válidos } & 454 & 100,0 \\
\hline
\end{tabular}

Método Estadístico. La estimación de la proporción de ocultación del hábito realizada sobre las mujeres que dijeron no fumar (ya que en ellas se determinó la cotinina) se llevó a cabo de manera puntual y por intervalo exacto de Clopper-Pearson. Aprovechando las mismas mujeres de las que se disponían de cifras de $\mathrm{CO}$, se determinó la calidad diagnóstica del mismo frente a la cotinina, "Gold Standard", mediante la curva ROC correspondiente, el área bajo la curva, estimada por el método del trapezoide, con su intervalo de confianza y estimando sensibilidad y especificidad para algunos puntos de corte ${ }^{20}$.

En todas las mujeres de nuestra muestra, 454, se disponía de la medida del CO y del auto reporte, no siendo ninguno de los dos un "Gold Standard"; sin embargo, para determinar la calidad diagnóstica de la autodeclaración para diferentes puntos de corte del CO se estimaron de manera puntual, y por intervalos con el procedimiento ya citado, la sensibilidad, la especificidad el valor predictivo positivo y el negativo de la autodeclaración para ser fumadora considerando como "Gold Standard" el CO. Este procedimiento es inverso al que se sigue usualmente por lo que sus resultados deben leerse con precaución.

\section{RESULTADOS}

\section{Proporción de ocultación/engaño. Descripción de los valores de cotinina obtenidos}

La medida de la cotinina se hizo en 104 mujeres fumadoras que declararon haber 
Figura 1

Curva ROC del CO en aire espirado para el diagnóstico del hábito tabáquico

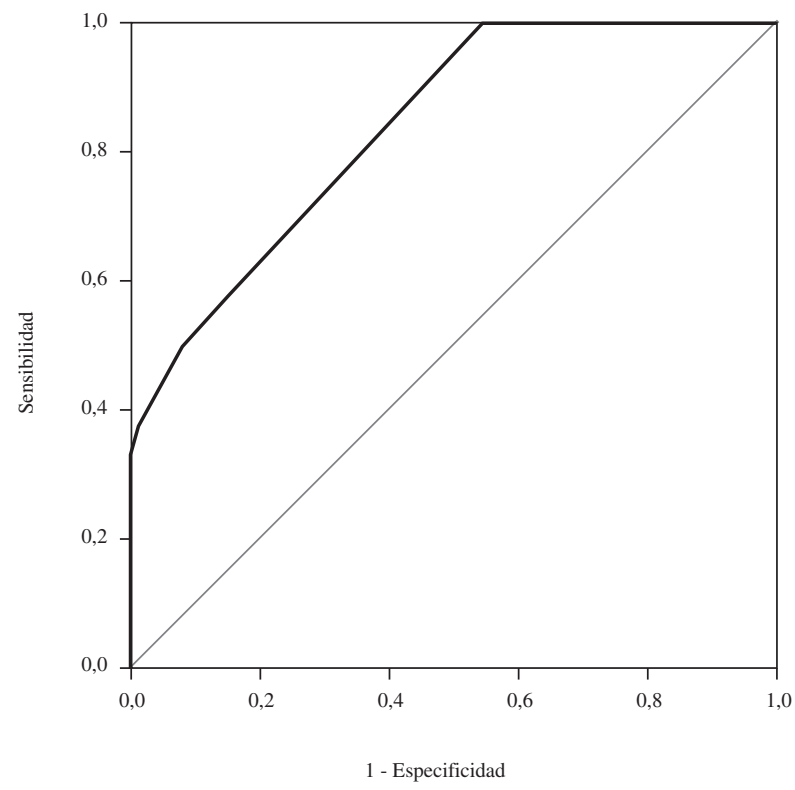

abandonado el hábito en la primera entrevista (abandono espontáneo). De entre esas mujeres, 16 , el $15,4 \%$, (IC al $95 \%$ 9,32-24,08), tenían una cotinina en orina superior a 100 lo que indicaría que eran fumadoras.

\section{Validez de la medición de $\mathrm{CO}$ en aire espirado}

En las 104 mujeres se disponía de la medida de cotinina y también de la medida de $\mathrm{CO}$ por lo que se determinó la curva ROC para dicha variable obteniéndose la figura 1. El área bajo la curva ROC resultó de 0,838, (IC 95\% 0,740-0,935).

De la curva ROC se obtuvo que para un punto de corte de 1 del $\mathrm{CO}$ la sensibilidad era del $100 \%$ y la especificidad de un $45,45 \%$; cuando pasamos a 2 cómo punto de corte del CO saltamos ya a una especifi- cidad de un $92,05 \%$ y a una sensibilidad de un $50 \%$; de otro lado si se usa el, clásico punto de corte, valor 9 de $\mathrm{CO}$ se consigue una especificidad de un $100 \%$ y una sensibilidad del $12,5 \%$.

\section{Autodeclaración y medición del CO}

Ya hemos visto en el primer apartado que su porcentaje de "errores" cuando se compara con la cotinina es de un $15,4 \%$, nos resta determinar de manera muy precisa la calidad diagnóstica de ese reporte para el caso de usar el CO como patrón.

De las 454 mujeres 132 (30,8\%) se declararon fumadoras. Considerando todas, la concentración de $\mathrm{CO}$ en aire espirado varió desde 0 p.p.m. a 48 p.p.m. con una media de 7,7 y una desviación típica de 8,31 ; la mediana resultó de 5 . La relación entre $\mathrm{CO}$ en aire espirado y la autode- 
Tabla 2

Medidas de validez de la autodeclaración considerando CO como patrón de referencia con diversos valores de corte

\begin{tabular}{|c|c|c|c|c|}
\hline \multicolumn{5}{|c|}{ Valor de corte en 1} \\
\hline \multirow{4}{*}{$\mathrm{CO}$} & \multicolumn{4}{|c|}{ Autodeclaración del habito de tabaco } \\
\hline & & No fuma & Si fuma & TOTAL \\
\hline & No fuma $(\mathrm{CO} \leq 1)$ & 50 & 14 & 64 \\
\hline & Si fuma $(\mathrm{CO}>1)$ & 81 & 282 & 363 \\
\hline Sensibilidad \% & Especificidad \% & Valor predictivo negativo $\%$ & Valor predictivo positivo $\%$ & Prevalencia fumadoras \\
\hline 77,7 & 78,1 & 38,2 & 95.3 & $85 \%$ \\
\hline \multicolumn{5}{|c|}{ Valor de corte en 2} \\
\hline \multirow{4}{*}{$\mathrm{CO}$} & \multicolumn{4}{|c|}{ Autodeclaración del habito de tabaco } \\
\hline & & No fuma & Si fuma & TOTAL \\
\hline & No fuma $(\mathrm{CO} \leq 2)$ & 108 & 39 & 147 \\
\hline & Si fuma $(\mathrm{CO}>2)$ & 23 & 257 & 280 \\
\hline Sensibilidad \% & Especificidad \% & Valor predictivo negativo $\%$ & Valor predictivo positivo $\%$ & Prevalencia fumadoras \\
\hline 91,8 & 73,5 & 82,4 & 86.8 & $65.6 \%$ \\
\hline \multicolumn{5}{|c|}{ Valor de corte en 9} \\
\hline \multirow{4}{*}{$\mathrm{CO}$} & \multicolumn{4}{|c|}{ Autodeclaración del habito de tabaco } \\
\hline & & No fuma & Si fuma & TOTAL \\
\hline & No fuma $(\mathrm{CO} \leq 9)$ & 130 & 172 & 302 \\
\hline & Si fuma $(\mathrm{CO}>9)$ & 1 & 124 & 125 \\
\hline Sensibilidad $\%$ & Especificidad \% & Valor predictivo negativo $\%$ & Valor predictivo positivo $\%$ & Prevalencia fumadoras \\
\hline 99,2 & 43,0 & 99,2 & 41.4 & $29.3 \%$ \\
\hline
\end{tabular}

claración existe de manera significativa $(\mathrm{P}<0,001)$. En la tabla 2 figuran los valores de los parámetros de validez de la autodeclaración para el caso del uso del CO como "Gold Standard" cortando en tres valores: 1,2 y 9 .

En el caso del punto de corte en 1, la sensibilidad del autoreporte es de un $77,7 \%$ lo que hace que el porcentaje de mujeres que ocultan su hábito en ese caso es de un $22,3 \%$, este porcentaje es ligeramente superior al porcentaje de ocultación con la cotinina que era de un $15,4 \%$ lo que nos indica que estaríamos sobrevalorando la ocultación; si se aumenta el punto de corte del $\mathrm{CO}$ a 2, habrá menos mujeres fumadoras y ese descenso, lógicamente, será mayor entre las que no fuman por lo que la sensibilidad aumentará a un $91,8 \%$ y la ocultación será ahora de un $8,2 \%$, lo que representa una subestimación de la ocultación; esta subestimación es mucho mayor cuando consideramos el, clásico, punto de corte en 9 para el que la sensibilidad da un $99,2 \%$ con lo que la estimación de la ocultación es de un $0,8 \%$ más de 15 veces inferior a la que hemos estimado frente al "Gold Standard" real.

\section{DISCUSION}

La autodeclaración de la gestante podría considerarse una de las variables más valiosas para establecer el hábito tabáquico debido a su simplicidad y al bajo coste, si no fuera por el fenómeno de la ocultación, descrito en la bibliografía, pero poco estudiado en nuestro medio. El único estudio publicado ${ }^{2}$ se hace con una muestra de 58 gestantes en primera consulta prenatal, donde 9 se declararon no fumadoras, dato confirmado sólo en 6 por la cotinina (30\% de ocultación). En la ultima visita prenatal la muestra sube a 108 , 32 con autodeclaración negativa de las cuales se confirman 28 con la cotinina 
(12.5\% de ocultación). No sabemos si las muestras son independientes y por tanto pudieran agruparse, ni si tiene sentido el calculo de una "ocultación media" en al gestación con este diseño. En el estudio que presentamos la proporción de ocultación del consumo de tabaco en las gestantes que se declaran no fumadoras en la primera visita es $15,4 \%$ (IC al 95\% 9,32-24.0). Esto significa, por ejemplo, que en la muestra en vez de un $30,8 \%$ de abandonos espontáneos al inicio del embarazo, tendríamos un 26, $2 \%$.

La trascendencia de este hecho es más epidemiológica que clínica, ya que es difícil, si no imposible, tratar a una persona dependiente que no reconoce u oculta su adicción. Sin embargo para el clínico es importante estar advertido de este fenómeno y sus posibles causas (relacionadas con la deseabilidad social del hábito). Un interrogatorio directo o culpabilizador, o incluso el uso de pruebas diagnosticas precisas para "desenmascarar" a la paciente aumentará el porcentaje de ocultación y romperá los posibles vínculos que faciliten el reconocimiento del problema y la posible ayuda del terapeuta. Por otro lado es clara la necesidad de incorporar las medidas de validación bioquímica de la autodeclaración a todos los estudios de prevalencia y de efectividad de las intervenciones para considerarlos de calidad aceptable.

En nuestro estudio la cotinina no se midió en mujeres que se etiquetaban a sí mismas como fumadoras y por tanto no se puede afirmar nada de las "equivocaciones en esos casos".El sentido común nos hace pensar que si hubiese algún caso seria mas lógico considerarlo un falso positivo del método de validación, ya que la fuerte presión social hace impensable que una no fumadora se declare fumadora.

Por ultimo en relación con la prevalencia de este fenómeno, queremos resaltar que con nuestros resultados nada podemos saber de los porcentajes de ocultación en situaciones no similares a la nuestra y sobre todo en otros momentos de la gestación. Tenemos la sensación de que el fenómeno de ocultación es dinámico y complejo y en cualquier caso el resultado de la interacción entre la gestante y los servicios de salud que la atienden. Esta seria una explicación de la gran variabilidad de los porcentajes de ocultación encontrados en diversas investigaciones. Probablemente requeriría de estudios longitudinales diseñados específicamente para su caracterización.

El segundo propósito era la validación del CO como método de confirmación bioquímica de la autodeclaración. En nuestra investigación hemos partido de tres premisas. La primera tiene que ver con la utilidad de la validación, que a nuestro juicio es la confirmación de la abstinencia en embarazadas que se declaran no fumadoras (asumimos que una embarazada es fumadora si se declara como tal), es decir no la usamos como método de diagnostico de tabaquismo activo, sino como identificación de ocultadoras. El diagnostico de tabaquismo en el embarazo se haría a través de la autodeclaración (si dice que fuma es verdad, considerándola la prueba mas sensible) y la validación bioquímica aplicada a las que dicen no fumar (si dice que no fuma hay que comprobarlo con una prueba muy especifica del hecho de no fumar). La segunda premisa es un corolario de este razonamiento: la población sujeta a estudio de estas investigaciones son las gestantes que se declaran no fumadoras. Esta afirmación tiene consecuencias transcendentes a la hora de escoger los procedimientos de análisis y tratamiento de los datos. Por ultimo, cualquier sistema de validación debe utilizar la medición de la cotinina como "patrón oro" de comparación y métodos estadísticos y epidemiológicos adecuados (calculo de área bajo la 
curva ROC, determinaciones de sensibilidad, especificidad y valores predictivos a distintos puntos de corte).

La hipótesis de que el $\mathrm{CO}$ es valido en nuestro medio queda confirmada al comprobar que el área bajo la curva ROC es de 0,838 (IC al 95\%, de 0,740 a 0.935). La utilidad de este método ya fue puesta de manifiesto por Seidman et $\mathrm{al}^{21}$ en un estudio observacional prospectivo donde aparecieron diferencias significativas de concentración de $\mathrm{CO}$ en el aire espirado en las gestantes que se declararon fumadoras activas $(n=17)$, fumadoras pasivas $(n=31)$ o no fumadoras $(n=20)$ : también se encontró correlación significativa entre el $\mathrm{CO}$ y la cotinina en sangre. Esta observación queda corroborada usando métodos epidemiológicos adecuados.

El punto de corte más idóneo para decidir quien es fumadora y quien no es el siguiente aspecto de los resultados que planteamos a discusión. Nuestros datos sugieren, que para la población gestante 9 no es el punto mas adecuado y que este debería ser mas bajo. En primer lugar la comparación con la cotinina nos muestra una sensibilidad de $12.5 \%$ en este punto, a todas luces insuficiente. La comparación

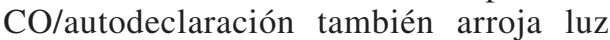
sobre este aspecto (Tabla 2). Hay que tener en cuenta que en esta tabla hemos variado los valores del $\mathrm{CO}$, el "Gold "Standard", justo al revés de lo que ocurre para una curva ROC en la que se mueven los puntos de corte del test diagnóstico frente al "Gold Standard" binario, este hecho hace que deba ser leída con precaución. Lo primero a observar es que conforme se aumenta el punto de corte del CO la estimación de la prevalencia del hábito tabáquico disminuye, cosa lógica porque habrá menos mujeres que cumplan la condición; al ser menor la prevalencia el valor predictivo positivo de la autodeclaración disminuirá ya que su dependencia de la prevalencia es directa y si este lo hace el valor predictivo negativo debe aumentar, porque hay menos mujeres con el hábito. Con esta advertencia, comprobamos que, en el punto de corte 9, la validez para la detección de ocultadoras de ambos métodos es similar ( $0.8 \%$ de falsos negativos) y la prevalencia de abandonos espontáneos sube hasta cifras irreales $(58,1 \%$ vs. $26 \%$ con la cotinina) .

Como dice Secker-Walker ${ }^{11}$, no hay ningún estudio, en el momento de realizar el suyo, que compare autodeclaración, cotinina y CO para clasificar a las gestantes como fumadoras. De las investigaciones realizadas que usan el $\mathrm{CO}$ como validación de la autodeclaración solamente dos, Hugues et al ${ }^{15}$ y Hartman et al ${ }^{14}$, determinan los puntos de corte con datos obtenidos de embarazadas fumadoras y no fumadoras. En el resto aplican puntos de corte referidos en la literatura, de poblaciones no embarazadas. El método usado para decidir sobre este punto ha sido el ajuste entre las distribuciones de $\mathrm{CO}$ y la autodeclaración. Es decir se tomaba el punto de la escala de $\mathrm{CO}$ que mejor discriminaba a las fumadoras y a las no fumadoras según la autodeclaración. Una verdadera tautología: pretender validar la autodeclaración usando como "gold Standard" la propia autodeclaración. No deja de sorprendernos que Secker-Walker poseyendo datos de cotinina utilice este procedimiento.

Como se ha comentado mas arriba, el resto de estudios utilizan puntos de corte referidos en la bibliografía para población no embarazada. Revisemos sus procedimientos de cálculo. En nuestro medio Becoña y Vázquez ${ }^{5}$ aconsejan en función de su experiencia un punto de corte de 9 p.p.m. En su publicación del 2004, Becoña y Lorenzo ${ }^{6}$ muestran una serie que relaciona el estatus de fumar (no fumador y número de cigarrillos fumados: status conocido por autodeclaración) con la media de la determinación de p.p.m de 
$\mathrm{CO}$ en los fumadores que acuden a tratamiento, oscilando los datos desde 2 p.p.m en no fumadores hasta 29 p.p.m en los fumadores de mas de 30 cigarrillos diarios. Frederiksen ${ }^{9}$ publica una serie de 15 no fumadores y 53 fumadores (desconocemos el procedimiento a través del cual conoce su estatus) donde en la primera los niveles de $\mathrm{CO}$ varían entre 2 y 8 (media de 4.93), mientras que en la segunda oscilan entre 6 y 90 (media de 34.4). Significativamente solo $2(4 \%)$ de los 53 fumadores se solapan en la distribución de no fumadores, pero 5 no fumadores $(30 \%)$ están incluidos en el rango de la distribución de fumadores. En esta serie apostar por la sensibilidad significaría decidirse por un punto de corte de 8 , por el contrario apostar por la especificidad bajaría el punto de corte hasta 5 . McMorrow et al 22 en su revisión manifiesta "que las medidas de CO correlacionan bien con otras medidas del estatus de fumador (autodeclaración) cuando se usan puntos de corte entre 5 y 10 ”. En nuestra opinión todos estos estudios y revisiones, salvo el de Frederiksen que tiene un escaso numero de casos, adolecen del problema comentado mas arriba: intentan validar la autodeclaración con una escala cuyo punto de corte se ha calculado partiendo de la autodeclaración como "gold Standard".

En cualquier caso el artículo más citado y seguido por los investigadores en estudios con gestantes fumadoras que usan el $\mathrm{CO}$ como método de validación es el de Jarvis et $\mathrm{al}^{23}$.

Jarvis presenta una muestra de 211 sujetos procedentes de las consultas externas de cardiología y cirugía vascular del St Mary's Hospital de Londres (159 hombres con media de edad de 56, 52 mujeres media de edad 55.3) en la que hay una prevalencia de tabaquismo activo a través de la autodeclaración de 43\% (121 no fumadores vs. 90 fumadores). De los 121 no fumadores 21 son identificados como ocultadores por la cotinina. A partir de este hecho construye el "gold Standard" sumando a los fumadores de la muestra los que se autodeclaran no fumadores pero presentan en sangre y orina cantidades apreciables de cotinina o nicotina. De esta forma obtiene para el $\mathrm{CO}$ un punto de corte de 8 que hace máxima la sensibilidad $(90 \%)$ y la especificidad (89\%). Nuestros resultados no son comparables con los de Jarvis ya que hay que considerar que él aplica el método sobre una población de fumadores y no fumadores, mientras que nosotros lo hacemos sobre una población que se autodeclara no fumadora para descubrir los casos de ocultación. Nuestra muestra esta sesgada en el sentido de que la proporción de fumadores es mucho menor, ya que hemos excluido los que se declaran fumadores. Por esta razón su punto de corte de sensibilidad y especificidad máxima se desplaza hasta el 8 . Nuestros resultados en ese punto difieren notablemente, con una especificidad del $100 \%$ conseguimos una sensibilidad del $12.5 \%$, con un comportamiento prácticamente similar a la autodeclaración. La pregunta es isi queremos identificar "ocultadores" sobre que población es mas legitimo construir el índice?

Es probable que otra causa de disparidad sea la no comparabilidad de las muestras. La de Jarvis es población mayor, preferentemente masculina y con patología crónica que probablemente afecta a la difusión pulmonar del CO y la nuestra es de jóvenes gestantes.

Mostrados los resultados de nuestra muestra hemos de precisar que presentan algunos problemas debido al tamaño con el que se ha trabajado. En efecto, aunque la muestra de mujeres a las que se les midió la cotinina es de 104, no fumadoras eran 88 , la muestra sobre la que se estima la especificidad, y 16 eran fumadoras, la muestra sobre la que se estima la sensibilidad. 
Ambas muestras son pequeñas, pero especialmente la de fumadoras, lo que implica que nuestras estimaciones son muy variables, dando lugar a intervalos de confianza anchos. Además tampoco es calculable el valor predictivo positivo ni el valor predictivo negativo del $\mathrm{CO}$, pues esta no es una muestra de todas las embarazadas sino sólo de las embarazadas que dicen que han dejado de fumar.

Todas estas reflexiones nos llevan a plantear una serie de consideraciones finales. La primera es que en la actualidad no hay pruebas suficientes para recomendar de forma taxativa un punto de corte de $\mathrm{CO}$ que discrimine adecuadamente entre gestantes fumadoras y no fumadoras.

La segunda es que si lo que pretendemos con el método es descubrir el fenómeno de ocultación/engaño, la población a estudio son las gestantes que se declaran no fumadoras y el punto de corte debería primar la capacidad para detectar verdaderos negativos (no fumadoras de verdad) con un coste de errores en la clasificación de fumadoras aceptable., nuestros datos sugieren que, al menos al inicio del embarazo, este debería ser bastante inferior a 9 .

Por ultimo todo ello plantea la necesidad de estudios más amplios de validación del $\mathrm{CO}$ en población gestante que se declara no fumadora con una metodología adecuada desde el punto de vista epidemiológico y estadístico. La posibilidad de variación a lo largo de la gestación sugiere la importancia de realizarlos en distintos momentos del embarazo.

\section{BIBLIOGRAFÍA}

1. Barrueco M, Jimenez Ruiz c, Palomo L, Torrecilla M, Romero P, Riesco JA. Veracidad de la respuesta de los fumadores sobre su abstinencia en las consultas de deshabituación tabáquica. Arch Bronconeumol. 2005; 41(3): 135-140.
2. Castellanos ME, Muñoz MI, Nebot M, Payá A, Rovira MT, Planasa S, Sanromà M, Carreras R. Validez del consumo declarado de tabaco en el embarazo. Aten Primaria. 2000; 9: 629-632.

3. Russell T, Crawford M, Woodby L. Measurements for active cigarette smoke exposure in prevalence and cessation studies: Why simple asking pregnant women isn't enough. Nicotine Tob Res. 2004; 6.supl 2: S141-S151.

4. Windsor RA, N. R. Boyd y C. T. Orleans. A metaevaluation of smoking cessation intervention research among pregnant women: improving the science and art. Health Educ Res., 1998; 13 (3): 419-438.

5. Becoña y Vázquez. Tratamiento del tabaquismo. Madrid: Dickinson; 1998.

6. Becoña E, Lorenzo C. Evaluación de la conducta de fumar, en Monografía Tabaco Adicciones. 2004; 16 supl 2: 201-226.

7. Haley NJ, Sepkovic DW, Hoffman D. Elimination of cotinina from body fluids: Disposition in smokers and non smokers. AJPH. 1989; 79:10461048 .

8. Jarvis MJ, Russell MAH, Saloojee Y Expired air carbon monoxide: A simple breath test of tobacco smoke intake. BMJ. 1980; 281: 484-485.

9. Frederiksen, Martin. Carbon Monoxide and smoking behaviours. Addict Behav. 1979 4:21-30.

10. Longo LD The biological effects of carbon monoxide on the pregnant woman, fetus and the newborn. Am J Obstet and Gynecol. 1977; 129: 69-103.

11. Secker-Walker R, Vacek P, Flynn B, Mead P. Exhaled carbon monoxide and urinary cotinina as measures of smoking in pregnancy. Addict Behav. 1997; 22 (5): 671-684.

12. Benowitz R. The use of biologic fluid samples in assessing tobacco smoke consumption. En Grabowski y Bell (Eds.), Measurement in the analysis and treatment of smoking behaviour. Rockville, MD: National Institute of Drug Abuse; 1983. p. 6-26.

13. Pechanek M. Review of techniques for measurement of smoking behavior. En Matarazzo, Herd, Miller y Weiss (Eds.), Behavioral health. A handbook of health enhancement and disease prevention. Nueva York: Wiley; 1984.p 729-754.

14. Hartmann K, Thorp J, Pahel-Short L, Koch M; A randomiced controlled trial of smoking cessation 
intervention in pregnancy in an academic clinic Obstet and Gynecol. 1996; 87: 621-626

15. Hughes JR, Epstein LH, Andrasik F, Neff DF, Thompson DS: Smoking and carbon monoxide levels during pregnancy Addict Behav. 1982; 7: 271-276.

16. Price JH, Krol RA, Desmond SM, Losh DP, Roberts SM, Snyder FF Comparison of three antismoking interventions among pregnant woman in an urban setting: A randomized trial. Psychol Rep. 1991; 68: 595-604.

17. Bauman KE, Bryan ES; Dent CW. The influence of observing carbon monoxide level on cigarette smoking by public prenatal patients AJPH. 1983; 73: 1089-1091.

18. Burling TA, Bigelow GE, Robinson JC, Mead AM. Smoking during pregnancy Reduction via objective assessment an directive advice Behav Ther. 1991; 22: 31-40.
19. Campbell, M. J. Cluster randomization in general (family) practice research. En Stat Methods Med Res. 2000; 9: 81-94.

20. Pepe, M.S. (2003). The Statistical Evaluation of Medical Tests for Classification and Prediction. New York, Oxford University Press.

21. Seidman D, Paz I,Merlet-Aharoni I, Vreman H, Stevenson D, Gale R. Non-invasive validation of tobacco smoke exposure in late pregnancy using end-tidal carbon monoxide measurements J Perinatol. 1999; 19 (5): 358-361.

22. McMorrow M, Foxx RM. Cigarette brand switching: Relating assessment strategies to the critical issues Psychol Bull. 1985; 98 (1):139159.

23. Jarvis MJ, Tunstal-Pedoe H, Feyerabend C, Vesey C, Saloojee Y Comparison of test used to distinguish smokers from non smokers AJPH. 1987; 77: 1435-1438. 